\title{
Auditoría integral a los procesos administrativos y financieros de pyme. Experiencias en el caso Cooperativa de Ahorro y Crédito "El sol de los Andes" (Ecuador)
}

\section{Comprehensive audit to the administrative and financial processes of smes. Experiences in the cooperative case of savings and credit "El sol de los Andes" (Ecuador)}

\author{
Ruiz Alvarado, Priscila Isabel ${ }^{1 *}$ \\ ${ }^{1}$ Universidad Católica de Cuenca \\ *pruiza@ucacue.edu.ec
}

DOI: https://doi.org/10.26871/killkana_social.v02i01.47

\begin{abstract}
Resumen
Las Cooperativas de Ahorro y Crédito (COAC) en el Ecuador, en la provincia y el cantón Cañar, han tenido un notable crecimiento. En los últimos cinco años, se ha evidenciado la apertura de un considerable número de ellas en la ciudad, lideradas por grupos o asociaciones indígenas del Cañar o del norte del país, generando desarrollo y crecimiento en las actividades productivas en la cabecera cantonal, así como en las comunidades campesinas. Para el presente estudio se consideró el caso de una de ellas, que para este artículo la denominaremos "Cooperativa de Ahorro y Crédito el "Sol de los Andes", ya que por la confidencialidad que conllevan los informes de auditoría no se puede revelar la identidad del ente auditado. La LOEPS estipula que es obligación de la COAC la realización únicamente de una Auditoría Financiera Anual, dejando a un lado la evaluación del cumplimiento de los objetivos, utilización de recursos, cumplimiento de leyes y regulaciones. La eficiencia de los mecanismos de control implementados por la entidad son criterios tan válidos como los financieros, lo cual se conseguirá con la realización de una auditoría integral al proceso de ahorros programados de la institución financiera; servirá como una herramienta de mejora y fortalecimiento, ya que se enfoca a cuatro aspectos fundamentales de accionar administrativo y financiero. Estos en muchos de los casos los directivos de las nacientes cooperativas desconocen, ocasionando el estancamiento o la desaparición de las mismas. Para la realización del presente trabajo se utilizará la metodología de una auditoría integral, que parte de un conocimiento preliminar de la institución a ser auditada, luego la planificación por componente, ejecución de la auditoría, comunicación de resultados a los directivos y personal de la entidad y el monitoreo de las recomendaciones.
\end{abstract}

Palabras clave: Auditoría integral, cooperativas, procesos administrativos y financieros.

\begin{abstract}
The Savings and Credit Units in Ecuador have experienced a remarkable growth, especially in the province and canton Cañar. Evidence shows that, in the last five years, a considerable number of them have been opened in the city led by indigenous groups or associations from Cañar or the north of the country. This has generated the development and growth of the productive activities in the cantonal capital, as well as in the rural communities. For the present study, one of the cases mentioned above has been considered which, for this study purposes will be called "Cooperativa de Ahorro y Credito "El Sol de los Andes "; since, due to the confidentiality related to audit reports, the identity of the audited entity cannot be revealed. The LOEPS (Acronym in Spanish for Organic Law of Popular and Solidarity Economy) stipulates that it is the COAC's (Acronym in Spanish for Savings and Credit Union) responsibility to carry out a single Annual Financial Audit, leaving aside the objectives, the compliance evaluation, the use of resources, the compliance with laws and regulations, as well as the efficiency of the control mechanisms implemented by the entity. These criteria are as valid as the financial ones. This will only be achieved by conduct an integral audit of the financial institution's programmed savings process, which will work as an improvement and strengthening tool that focuses on four fundamental aspects of administrative and financial action. In many cases, these aspects are unknown by the new cooperatives' managers; causing therefore their stagnation or disappearance. In order to carry out the present study, an integral audit methodology will be used. It starts with the preliminary study of the institution to be audited, then comes the planning by component, after that, the performance of the audit takes place, followed by the reporting of results to the entity's managers and staff. The final step consists on monitoring the recommendations.
\end{abstract}

Key words: Integral audit, credit units. administrative and financial processes.. 


\section{Introducción}

Luego de la crisis económica vivida en la década de los noventa, el fenómeno de la migración se agudizó más en la provincia y ciudad de Cañar, llegando a los habitantes de las comunidades campesinas que en busca de mayores oportunidades de trabajo e ingresos para sus familias, dejaron sus hogares y emigraron a países como EEUU y España. Por otro lado cabe destacar la gran expansión de las COAC de los sectores rurales, tanto de la Zona Centro Norte como de la región Sur de la serranía ecuatoriana. Desde el 2008 con la nueva Constitución Política del Ecuador, a dado lugar a que en la ciudad de Cañar se incremente de manera considerable el número de Instituciones Financieras de este tipo. En este contexto, la Cooperativa de Ahorro y Crédito "El Sol de los Andes" es una de las nuevas instituciones que se han iniciado sus actividades en la ciudad de Cañar, para cumplir con objetivos comunes de superación con base en valores como la cooperación y la solidaridad bajo el liderazgo de 15 migrantes. Fue así como la cooperativa vio la luz en la ciudad de Cañar a los 31 días del mes de mayo del 2011 y desde esa fecha ha ido creciendo y ajustándose a los cambios en cuanto a las leyes, regulaciones y condiciones del entorno a las cuales se ha enfrentado.

La institución financiera pretende fomentar el desarrollo sostenible de sus asociados bajo el amparo y el fortalecimiento de la Economía Popular y Solidaria, promoviendo los principios de una cultura de excelencia que redunde en el desarrollo en procura del Buen Vivir (Sumak - Kawsay), y construyendo un nuevo modelo de gestión con atención integral a la sociedad intercultural.

El desconocimiento de los procesos y la utilidad de la realización de una auditoría integral por parte de los directivos de las cooperativas, y la exigencia de realizar de manera obligatoria una auditoría financiera anual, ocasiona que se realicen solamente este tipo de evaluaciones por cumplir con los requerimientos legales de los organismos de control. La realización de una evaluación a los cuatro componentes les permite obtener información más completa para una correcta toma de decisiones y acciones correctivas para la obtención de resultados más significativos para la entidad.

En el presente estudio se pretende aplicar un examen especial de auditoría integral al proceso de ahorros programados de la Cooperativa de Ahorro y Crédito "El Sol de los Andes", durante el año 2013, utilizando una metodología apegada a la normativa vigente en el Ecuador para este tipo de auditorías, con la finalidad de emitir un informe que sirva de herramienta de mejora continua para la institución.

Mediante la aplicación de esta auditoría se pretende dar respuesta a la interrogante ¿La aplicación de una auditoría integral dotara de una herramienta de mejora continua en los ámbitos financiero, de control interno, de gestión, y de cumplimiento que le permita a la cooperativa la correcta toma decisiones que se evidencien en el crecimiento y desarrollo sostenible de la entidad?
Por el crecimiento que se ha evidenciado desde su creación se hace necesario la aplicación de una auditoría integral para determinar si ese crecimiento es sostenido y sustentable, mediante la aplicación de este tipo de examen se obtiene una seguridad razonable del manejo eficiente de los recursos financieros, el cumplimiento de las leyes y regulaciones, a través de una administración con una orientación social y de resultados, los mismos que pueden ser cumplidos con un eficiente sistema de control interno, y la capacidad de cumplir los objetivos empresariales con la utilización racional de los recursos disponibles, la suma de estos factores hace que las organizaciones alcancen sus objetivos.

Al inicio de la investigación se presentaron algunas dificultades por cuanto los funcionarios de la cooperativa se resistían a brindar la información necesaria para el proceso de la auditoría, para solventarlas se programó una entrevista con los directivos y funcionarios implicados en la cual se les planteó las ventajas que tendría la entidad con la aplicación de este tipo de estudio, concluyendo con la apertura de parte de los directivos y funcionarios para la ejecución y conclusión de la investigación.

Con el presente artículo se pretende articular la docencia en la cátedra de auditoría y la puesta en práctica de los conocimientos impartidos en la misma, ya que existe una gran cantidad de bibliografía extrajera, pero la ampliación a un caso concreto del entorno cercano no es muy común.

\section{Marco teórico referencial}

\subsection{Definición de Auditoría}

En la actualidad la auditoría se ha convertido en una herramienta importante para la gestión de cualquier empresa, es por esa razón que nace la necesidad de evaluar a una empresa en otros aspectos a más del financiero, por lo tanto, se define a la auditoría integral como:

El proceso de obtener y evaluar objetivamente, en un período determinado, evidencia relativa a la siguiente temática: la información financiera, la estructura de control interno financiero, el cumplimiento de las leyes y regulaciones pertinentes y la conducción ordenada en el logro de las metas y objetivos propuestos; con el propósito de informar sobre el grado de correspondencia entre estos temas y los criterios o indicadores establecidos para su evaluación(Blanco, 2012)

Con base en la definición expuesta anteriormente se puede colegir que los objetivos que persigue la auditoría integral son:

- Emitir una opinión acerca de la razonabilidad de los resultados revelados en los estados financieros. y la concordancia con la normativa vigente en el área contable financiera.

- Determinar el cumplimiento de las normas legales, reglamentarias y estatutarias vigentes en el desarrollo de cada una de las actividades que realizan dentro de la organización. 
- Establecer el grado de eficiencia, eficacia y economía con el que se han conducido las actividades en pos del cumplimiento de los objetivos empresariales.

Evaluar el nivel de confianza en el diseño e implementación del sistema de control interno global.

\subsection{Auditorías que Conforman la Auditoría Integral.}

\subsubsection{Auditoría de Control Interno}

Definición de Control Interno: El control interno es un proceso, ejecutado por el consejo de directores, la administración y otro personal de una entidad, diseñado para proporcionar seguridad razonable con miras a la consecución de objetivos en las siguientes categorías:

- Efectividad y eficiencia de las operaciones.

- Confiabilidad en la información financiera.

- Cumplimiento de las leyes y regulaciones aplicables. (Mantilla B, 2005)

Componentes del Control Interno: Según el informe COSO I, descrito en la obra, "Control Interno, Informe COSO” de Samuel Alberto Mantilla B, un sistema de control interno tiene 5 componentes que se anotan en el grafico 1:

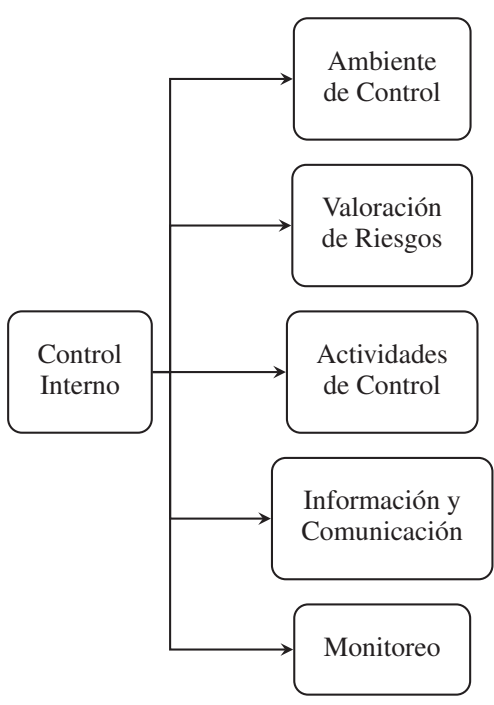

Figura 1. Control interno

Fuente: (Mantilla B, 2005)

\section{Objetivos de la Auditoría de Control Interno}

- Evaluar el sistema de control interno para comprobar si los controles establecidos por la empresa están operando de forma efectiva o consistente durante todo el ejercicio.

- Determinar el nivel de confianza a través de la eficiencia y eficacia al momento de cumplir con sus objetivos.

- Emitir una opinión acerca del sistema de control interno, sobre la calidad, oportunidad y validez de los controles establecidos por la administración. (Mantilla B, 2005)

\section{Alcance de la auditoría de control interno}

El alcance de una auditoría de control interno consiste en evaluar los controles implementados por la organización en los diferentes procesos o ciclos transaccionales durante el periodo auditado.

\subsubsection{Auditoría Financiera}

Definición de Auditoría Financiera:Es un examen sistemático de los estados financieros, registros y operaciones con la finalidad de determinar si están de acuerdo con los principios de contabilidad generalmente aceptados, con las políticas establecidas por la dirección y con cualquier tipo de exigencias legales o voluntariamente aceptadas. La auditoría tiene por objeto averiguar la exactitud, integridad y autenticidad de los estados financieros, expedientes y demás documentos administrativos-contables presentados por la dirección, así como sugerir las mejoras administrativocontable que proceda. (Madariaga, 2004).

\section{Objetivos de la Auditoría Financiera.}

- Examinar los estados financieros de una entidad para determinar su concordancia con los principios de contabilidad generalmente aceptados.

- Determinar la exactitud autenticidad e integridad de los estados financieros expresados en los documentos contables preparados por la administración.

- Emitir una opinión acerca de la razonabilidad de los resultados revelados en los estados financieros en cuanto a su estructura y cumplimiento de los principios y normas aplicables.

\section{Alcance de la Auditoría Financiera}

La auditoría financiera centra su atención en las afirmaciones que hacen los administradores de la organización auditada, con respecto a los datos presentados en los estados financieros en relación a las cuentas de orden o nominales y de balance o reales, aseverando así su correcta presentación, a través de las 4 afirmaciones: la Veracidad, Integridad, Valuación Exposición (VIVE).

\subsubsection{Auditoría de Cumplimiento.}

Definiciones de la auditoría de cumplimiento: La Auditoría de Cumplimiento es la comprobación o examen de las operaciones financieras, administrativas, económicas y de otra índole de una entidad, para establecer que dichas operaciones se han realizado conforme a las normas legales, reglamentarias, estatutarias y de procedimientos que le son aplicables. (Blanco, 2012)

Objetivos de la auditoría de cumplimiento.

- Verificar el cumplimiento de las leyes de carácter general y específico en el desarrollo de las actividades, operacionales, financieras, administrativas, y de otra índole que le sean aplicables.

- Verificar el cumplimiento de las normas legales, estatutarias y regulaciones internas en el desarrollo de las actividades de la empresa.

- Emitir un informe que contenga las inconformidades con la finalidad de tomar medidas correctivas en la organización. 


\section{Alcance de la auditoría de cumplimiento.}

Determinar si la sociedad en el desarrollo de las operaciones que realiza cumple con las disposiciones legales que le son aplicables.(Blanco, 2012)

\subsubsection{Auditoría de Gestión.}

Definiciones de Auditoría de Gestión: La auditoría de gestión es el examen que se efectúa a una entidad por un profesional externo e independiente, con el propósito de evaluar la eficacia de la gestión en la relación con los objetivos generales; su eficiencia como organización y su actuación y posicionamiento desde el punto de vista competitivo, con el propósito de emitir un informe sobre la situación global de la misma y la actuación de la dirección. (Blanco, 2012)

\section{Objetivos de la Auditoría de Gestión.}

- Determinar el grado de cumplimiento de los objetivos a corto, mediano y largo plazo, mediante la aplicación de indicadores de desempeño.

- Establecer el grado de eficiencia, eficacia y efectividad de las actividades empresariales, así como su impacto en el entorno.

- Identificar las oportunidades que se presenten para mejorar la gestión y minimizar las debilidades.

- Emitir un informe que contenga los hallazgos encontrados durante la auditoría, así como conclusiones y recomendaciones.

\section{Alcance de la Auditoría de Gestión}

La auditoría de gestión se centra en la evaluación del grado de cumplimiento de los objetivos y la cantidad de recursos empleados, es decir la eficiencia y eficacia en el logro de los objetivos planificados por empresa y el manejo de los recursos disponibles.

\subsection{Proceso de la Auditoría integral.}

Al igual que las demás auditorías la integral sigue una metodología que interrelaciona una serie de procesos hasta llegar a cumplir su objetivo, las fases se anotan en la tabla1.

\section{Metodología}

La metodología utilizada en el presente trabajo fueron los métodos de análisis, síntesis y deducción. mediante la aplicación de técnicas y procedimientos de auditoría sobre una muestra significativa de las operaciones realizadas en el Proceso de Ahorros Programados de la Cooperativa de Ahorro y Crédito "El Sol de los Andes" en cada una de las fases de la auditoría integral descritas anteriormente en la tabla $\mathrm{N}^{\circ}$ 1, (Fases de la Auditoría Integral), necesarias para la obtención de evidencia suficiente y competente, que respaldan la opinión acerca de los resultados obtenidos durante el examen de auditoría integral.

\subsection{Resultados de auditoría integral}

Se realiza un examen especial de auditoría integral al Proceso de Ahorros Programados de la Cooperativa de Ahorro y Crédito "El Sol de los Andes", del periodo comprendido entre el 1 de enero al 31 de diciembre de 2013, que cubre un examen al Estado de Situación Financiera, Estado de Resultados, Estado de Cambios en el Patrimonio Neto y Estado de Flujo de Efectivo; la evaluación del sistema de control interno; la evaluación del cumplimento de las leyes y regulaciones que afecta el grado de eficiencia y efectividad en el manejo de sus programas y actividades medidas a través de indicadores de gestión. El examen de auditoría integral se realiza de acuerdo con las Normas de Auditoria Generalmente Aceptadas, Normas Internacionales de Auditoría y Normas Ecuatorianas de Auditoría, y a la normativa vigente en el Ecuador para el Sistema Financiero al cual pertenece la entidad, en cada una de las clases de auditoría contempladas dentro de la auditoría integral, mismas que establecen que el trabajo debe ser planificado y ejecutado en función de la obtención de una seguridad razonable que los Estados Financieros no contengan errores significativos, de igual manera en cuanto a si la estructura del Sistema de Control Interno ha sido diseñado adecuadamente y opera de manera efectiva; el grado de cumplimiento de las leyes y regulaciones vigentes en el país y en el sector al que pertenece la institución auditada, al tratarse de una auditoría integral se evaluó también el grado de eficiencia, eficacia, y calidad de su Planificación Operativa Anual por medio de indicadores de gestión.

\subsubsection{Comentarios de auditoría financiera}

\section{Diferencia en declaraciones}

Se ha determinado una diferencia en la declaración de retenciones en la fuente del mes de abril por retenciones en rendimientos financieros se tiene, $\$ 38,93$ la cual se encuentra con su correspondiente retención del $2 \%$, valor que fue descontado al depositante, pero no fue presentado en la declaración de abril; Según el Art. 83, inciso 1 de la constitución de la república del Ecuador, expresa la obligatoriedad del cumplimiento de la constitución y leyes que rigen en el estado Ecuatoriano, también se tiene que verificar el Art. 50 Obligaciones de los agentes de retención. En su parte pertinente manifiesta, “están obligados a declarar y depositar mensualmente los valores retenidos en las entidades legalmente autorizadas para recaudar tributos, en las fechas y en la forma que determine el reglamento", se debe considerar además El Art 153 en su parte pertinente manifiesta la máxima autoridad del sujeto activo correspondiente, podrá conceder para el pago de esa diferencia plazos hasta de dos años. Es importante tener presente también la NIA 11; el incumplimiento se debe al inadecuado control interno en la generación oportuna de la documentación provocando información deficiente e incompleta entregada al contador de la organización por parte del encargado de pagos; Lo que ocasiona la exclusión de beneficios tributarios, problemas legales con el estado e 
Tabla 1 Fases de la auditoria integral

\begin{tabular}{|c|c|c|c|c|}
\hline FASES & OBJETIVO & ALCANCE & DOCUMENTACIÓN DE ENTRADA & PRODUCTOS \\
\hline $\begin{array}{c}\text { FASE I } \\
\text { PLANIFICACIÓN } \\
\text { PRELIMINAR }\end{array}$ & $\begin{array}{l}\text { Obtener un conocimiento } \\
\text { general de la entidad a ser } \\
\text { auditada, con la finalidad } \\
\text { de determinar el objetivo } \\
\text { y alcance de la auditoría. }\end{array}$ & $\begin{array}{l}\text { Conocimiento } \\
\text { de la base } \\
\text { estructural } \\
\text { conceptual } \\
\text { y legal de la } \\
\text { empresa }\end{array}$ & $\begin{array}{l}\text { Estatutos, Manuales, Orgánico Funcional, Le- } \\
\text { yes y Reglamentos del sector, Planificación de } \\
\text { actividades entre, plan de cuentas, reportes de } \\
\text { producción, vetas e inventarios. }\end{array}$ & $\begin{array}{l}\text { Informe de } \\
\text { planificación } \\
\text { específica } \\
\text { diagnóstico }\end{array}$ \\
\hline $\begin{array}{c}\text { FASE II } \\
\text { PLANIFICACIÓN } \\
\text { ESPECÍFICA }\end{array}$ & $\begin{array}{l}\text { Diseñar planes de audito- } \\
\text { ría específicos }\end{array}$ & $\begin{array}{l}\text { Elaboración de } \\
\text { los programas } \\
\text { de auditoría. }\end{array}$ & $\begin{array}{l}\text { Información en la que se reflejen procesos } \\
\text { importantes }\end{array}$ & $\begin{array}{l}\text { Planes y Pro- } \\
\text { gramas de tra- } \\
\text { bajo a la medi- } \\
\text { da. }\end{array}$ \\
\hline FASE III EJECUCIÓN & $\begin{array}{l}\text { Evidenciar la aplicación } \\
\text { de los programas de tra- } \\
\text { bajo }\end{array}$ & $\begin{array}{l}\text { Aplicación de } \\
\text { los programas } \\
\text { de trabajo a la } \\
\text { medida }\end{array}$ & $\begin{array}{l}\text { Programas de trabajo. Cronograma de ejecu- } \\
\text { ción de la auditoría. }\end{array}$ & $\begin{array}{l}\text { Documentación } \\
\text { de la ejecución } \\
\text { y Archivo } \\
\text { Corriente }\end{array}$ \\
\hline $\begin{array}{c}\text { FASE IV } \\
\text { COMUNICACIÓN DE } \\
\text { RESULTADOS }\end{array}$ & $\begin{array}{l}\text { Comunicar los hallazgos } \\
\text { encontrados durante la } \\
\text { ejecución con el propó- } \\
\text { sito de impulsar acciones } \\
\text { correctivas de inmediato }\end{array}$ & $\begin{array}{l}\text { Comunicación } \\
\text { parcial y } \\
\text { definitiva de } \\
\text { los resultados } \\
\text { con proyección } \\
\text { a identificar } \\
\text { oportunidades } \\
\text { de mejora }\end{array}$ & $\begin{array}{l}\text { Programas de trabajo. Papeles de trabajo. } \\
\text { Apuntes realizados por los auditores }\end{array}$ & $\begin{array}{l}\text { Informe de au- } \\
\text { ditoría integral }\end{array}$ \\
\hline $\begin{array}{c}\text { FASE V } \\
\text { SEGUIMIENTO }\end{array}$ & $\begin{array}{l}\text { Diseño de recomenda- } \\
\text { ciones que deberán ser } \\
\text { implementadas luego de } \\
\text { concluida la auditoría }\end{array}$ & $\begin{array}{l}\text { Diseño de un } \\
\text { plan de imple- } \\
\text { mentación de } \\
\text { recomendacio- } \\
\text { nes }\end{array}$ & $\begin{array}{l}\text { Informe ejecutivo y las observaciones realiza- } \\
\text { das por los involucrados, los compromisos y } \\
\text { las opiniones para la mejora de las debilidades } \\
\text { encontradas. }\end{array}$ & $\begin{array}{l}\text { Matriz de im- } \\
\text { plementación } \\
\text { de recomenda- } \\
\text { ciones }\end{array}$ \\
\hline
\end{tabular}

Fuente:(Blanco, 2012)

información financiera con errores significativos que pueden alterar la razonabilidad de los estados financieros.

\subsubsection{Comentarios de auditoria de control interno}

\section{Pólizas incompletas}

Del estudio realizado se desprende que el $47 \%$ de las pólizas examinadas carecen de firmas de la Gerente o del socio o el emisor del documento; Al momento de realizar la captación la persona que registre una póliza en el sistema tiene la obligación de imprimir el documento y solicitar las firmas de legalización al socio, gerente y emisor; esta situación obedece a la falta de organización en el proceso y responsabilidad del mismo. Los documentos pueden carecer de valor legal, y pueden ocasionar problemas al no existir un responsable de la transacción.

\section{Inexistencia de un responsable}

De la evaluación realizada se desprende que no existe un departamento ni una persona responsable del proceso de ahorros programados o depósitos a plazo en la cooperativa; la Ley Orgánica de la Economía Popular y Solidaria y del Sector Financiero y Popular en el artículo 83 Actividades Financieras, literal a) dice "Recibir depósitos a la vista y a plazo, bajo cualquier mecanismo o modalidad autorizado", al ser una actividad innata de cualquier Institución del Sistema Financiero debe tener un responsable para seguridad y salvaguarda del dinero de los socios; esto obedece a la inadecuada organización, asignación y segregación de funciones y responsabilidades; lo que ocasiona el incumplimiento de las políticas y requisitos en la negociación y legalización de las pólizas.

\section{La cooperativa no cuenta con la clave uaf}

Durante el proceso de la auditoría se pudo determinar que la cooperativa no cuenta con la clave UAF; en la Ley Orgánica de la Economía Popular y Solidaria y del Sector Financiero y Popular en el artículo 93 Prevención de Lavado de Activos estipula " Las Cooperativas de ahorro y Crédito implementaran mecanismos de prevención de lavado de activos conforme a las disposiciones constantes vigentes....", no se ha logrado obtener la clave por la inadecuada gestión por parte de los dierctivos de la Cooperativa; por lo tanto existe el riesgo de que ingrese dinero de dudosa procedencia, y no se cumpla con los reportes a la Unidad de Análisis Financiero.

\subsubsection{Comentario de auditoria de cumplimiento}

Incumplimiento en la aplicación de las tasas de interés para los depósitos a plazo.

De la evaluación realizada a las pólizas existentes durante el año dos mil trece se observa el incumplimiento en la aplicación de las tasas de interés de la muestra análizada solamente el 17,64\% de las pólizas aplican las tasas que le corresponden; de acuerdo a lo autorizado por las autoridades en función del tiempo y el monto, lo que afecta a los saldos de las cuentas de intereses pagados, asi como tambien a la credibilidad de la cooperativa por parte de sus socios.

\subsubsection{Comentarios de auditoria de gestión}

Inadecuada planificación y ejecución del presupuesto de la cooperativa

Durante el año 2013 los ingresos obtenidos frente a los planificados se ejecutaron en un $121,87 \%$, por encima de lo planificado; en lo que respecta a los gastos presupuestados 
frente a los ejecutados se determina que la cooperativa gastó un $15,05 \%$ por encima de lo planificado, de los dos rubros tenemos una variación neta del 7,06\%; De acuerdo al Reglamento de la Ley de Economía Popular y Solidaria en los Artículos 44 y 34 en sus literales 3 y 12 respectivamente se mencionan que las Instituciones bajo su control deben contar con el presupuesto así como los documentos estratégicos de planificación aprobados por el Consejo de Administración para el siguiente año antes de culminar el ejercicio fiscal en ejecución, sin embargo esta variación se encuentra dentro de los límites aceptables establecidos por el ente de control; Esta diferencia se desprende de la falta de preparación en la rama de los responsables del proceso, y a la falta de control por parte del órgano competente; lo que provocaría una disminución en los ingresos e incremento en los gastos lo que ocasiona un incremento en las pérdidas acumuladas que la cooperativa presenta en sus ejercicios desde el 2012.

Inadecuada declaración de objetivos formales y mensurables, para el proceso de captaciones por ahorros programados.

Luego de revisada la documentación entregada por la cooperativa no existe una declaración de objetivos formales y claros sobre el proceso de ahorro programado; En el plan operativo al mencionar sus estratégias para el desarrollo, en el Proposito de la cooperativa, habla del "fortalecimiento del sistema de ahorro y crédito, fomentando el ahorro de los socios........"; Se debe a la falta de experiencia de los directivos y administradores en el manejo de instituciones finacieras; lo que puede repercutir en la disminución de los recursos para la colocación.

\section{Cursos o talleres planificados y realizados}

En el año 2013 se realizaron 4 cursos o talleres en lugar de los 6 planificados es decir cumplio el 66,67\% con una inversión de $\$ 1200,00$. La falta de organización al momento de ejecutar las actividades planificadas; ocasionó que el personal no participe en los cursos para mejorar su desempeño laboral.

\section{Conclusiones}

Es una cooperativa relativamente nueva en el mercado, y quienes están al frente tienen escaza experiencia en el ámbito financiero. El personal que labora a excepción del contador no tiene preparación en el área económica, administrativa o afines, su perfil tiene relación directa con la educación. No existe una segregación de funciones definidas en el proceso de ahorros programados, el proceso lo realizan el responsable de atención al socio, la gerente, la cajera o el presidente de la cooperativa de acuerdo a la disponibilidad de tiempo. No existe un manual ni un reglamento que regule las actividades en el proceso de ahorros programados. Existe la disposición del personal a ajustarse para desarrollar de mejor manera sus actividades.

En base a los hallazgos obtenidos en la auditoría integral realizada se desprende que la realización de este tipo de estudios fortalece la gestión de la cooperativa en todos los niveles y ámbitos de acción de la Cooperativa, ya que brinda una guía para mejorar continuamente, lograr los objetivos institucionales y cumplir con la normativa vigente.

\section{Recomendaciones}

Realizar cursos o talleres para directivos y el personal sobre la legislación vigente, liderazgo, organización y planificación. Realizar un plan de capacitación para el personal de acuerdo al área en temas como: Atención al socio, planificación y organización, manejo de herramientas informáticas aplicadas al área de trabajo.

Conformar el área o unidad de inversiones en la cooperativa y determinar un responsable del proceso, y separar las funciones de quien negocia y quien recibe el dinero. Elaborar un manual y reglamento para la operación del proceso de ahorros programados o depósitos a plazo, para que sus actividades se desarrollen de manera planificada, organizada y orientada hacia objetivos concretos y medibles. Se recomienda a discreción de los directivos el cumplimiento de estas y las sugerencias contenidas en el informe de auditoría integral.

En todas las cooperativas que pertenecen a este sector sería de mucha utilidad la realización de auditorías integrales por parte de los entes reguladores con la finalidad de proporcionarles una herramienta de gestión objetiva y mensurable en los cuatro aspectos fundamentales de su accionar.

\section{Referencias Bibliográficas}

Bacallao Horta, M. (2009, mayo). Génesis del Control Interno. Contribuciones a la Economía.

Beltrán Jaramillo, J. M. (2010). Indicadores de Gestión. En J. M. Beltrán Jaramillo (Ed.), Indicadores de Gestión (pp. 35-36). Bogotá: 3REditores.

Blanco, L. Y. (2012). Auditoría Integral Normas y Procedimientos. Bogota, COLOMBIA: ECOE EDICIONES.

Cook, J. W., y Whinkle, G. M. (1996). Auditoría. En J. W. Cook y G. M. Whinkle (Eds.), AUDITORÍA (p. 5). México: MCGraw-Hill Interaméricana.

Espinoza Arízaga, M. L. (2013). Auditoría de Cumplimiento I. En M. L. Espinoza Arízaga (Ed.), Auditoría de Cumplimiento I (pp. 21-23). Loja: Ediloja.

Franklin, E. B. (2007). Auditoría Administrativa. En E. B. Franklin (Ed.), Auditoría Administrativa Gestión estrátegica del cambio (p. Glosario 829). Naucalpan de Juarez, Edo. de México: Pearson Educación.

González, M. W. V. (2014). Auditoría de gestión 6. Loja, Loja, Ecuador: Ediloja.

Guzmán, C. A. (2006). Guía rápida ratios financieros y matemáticas de mercadotecnia. Prociencia y Cultura.

Instituto Mexicano, C. P., y Comisión de Normas y, P. d. A. (1996). Normas y Procedimientos de Auditoría. En 
C. P. Instituto Mexicano y P. d. A. Comisión de Normas y (Eds.), Normas y Procedimientos de Auditoría (pp. 1010-5). México DF: Veromart.

León Cornejo, M. (2012). Auditoría Financiera. En M. León Cornejo (Ed.), El Proceso de la Auditoría Financiera (p. 17). Loja: Ediloja.

Luna, Y. B. (2012). Auditoría integral. Bogotá: Ecoe Ediciones.

Madariaga, J. M. (2004). Manual Práctico de Auditoría. En J. M. Madariaga (Ed.), Manual Práctico de Auditoría (p. 13). Barcelona: Ediciones.

Mantilla B, S. A. (2005). Control Interno. En S. A. Mantilla B (Ed.), Control Interno, Informe COSO (p. 14). Bogotá: Ecoe ediciones.

Newton Fowler, E. (2004). Tratado de Auditoría. En E. Newton Fowler (Ed.), Tratado de Auditoría. Buenos Aires: La Ley.
Pombo, J. R. (2004). Contabilidad General. ThomsonParaninfo.

Sáchez, P. Z. (2011). Contabilidad General Con Base en las Normas Internacionales de Información Financiera. Bogotá: Mc.Graw Hill.

Sánchez Curiel, G. (2006). Auditoría de Estados Financieros. En G. Sánchez Curiel (Ed.), Auditoría de Estados Financieros Práctica Moderna Integral. México: Pearson Educación.

Sotomayor, A. A. (2008). Auditoría Adminstrativa. En A. A. Sotomayor (Ed.), Auditoría Adminstrativa (p. 33). México, DF: McGraw-Hill.

Whittington, O. R., y Pany, K. (2000). Auditoría: un Enfoque Integral. En O. R. Whittington y K. Pany (Eds.), Auditoría: un Enfoque Integral (p. 598). Bogotá: Mc. Graw Hill.

Recibido: 20 de octubre de 2017

Aceptado: 24 de enero de 2018 
